\title{
QUALITY MANAGEMENT IN SURGICAL DISCIPLINES
}

Georgios I. Tagarakis ${ }^{1,2}$ Costas Dikeos $^{1}$, Fani Tsolaki ${ }^{2}$, Marios Daskalopoulos ${ }^{2}$, Petros Bougioukakis ${ }^{3}$, Vasilis Haralaboboulos ${ }^{4}$, Nikolaos Tsilimingas ${ }^{2,}$ Nikolaos Polyzos ${ }^{1}$

1-Department of Social Administration and Political Science, Democritus University of Thrace

2-Department of Cardiovascular and Thoracic Surgery, University of Thessaly, Larissa, Greece

3-Department of Cardiac Surgery, Bad Neustadt a.d. Saale, Germany

4-Stirling Management, School, University of Stirling, Scotland, UK

Corresponding author: GI Tagarakis, MD, MSc, PhD, tel-+306970806717, e-mail: gtagarakis@gmail.com

\section{ABSTRACT}

Clinical protocols and the related clinical indicators of quality and performance are expressions of Evidence- based Medicine. They act as medical as well as managerial tools whose aim is to provide the best possible health care at the least possible cost, taking into account the means available in each circumstance. Herein we present two of the protocols used in our institutions, regarding lung tissue resection for lung cancer and inguinal hernia plastic surgery.

Key Words: quality management, clinical protocols, clinical indicators of quality, surgical disciplines

\section{Council for Innovative Research}

Peer Review Research Publishing System

Journal: Journal of Social Sciences Research

Vol .7, No.2

jssreditor.cir@gmail.com

www.jssronline.com 


\section{INTRODUCTION}

In continuation to our previous article ${ }^{1}$ regarding the significance of clinical protocols in surgical disciplines, we would like to present the next two of our protocols, designed after meticulous study of the related literature and after the evaluation of worldwide experts' opinion on the subject ${ }^{2-12}$. We would like to emphasize again the fact that, clinical protocols act both as a medical as well as a managerial tool, aiming at the improvement of clinical services provided to our patients, with the application of the means available each time at the least possible cost The rationale behind is that since health treatments have a cost and resources are limited, the integration of managerial and financial efficiency within clinical efficiency is crucial ${ }^{13,14,15,16}$. As Bachtsevani et al. ${ }^{14}$ put it, "it seems the use of evidence-based guidelines can improve outcomes in relation to organizations in the sense of decreased admission rates, length of stay, and less resource utilization, which reduces costs". Such monitoring mechanisms intend to evaluate, reassess and improve the clinical care not only in terms of outcomes but also in financial terms for the benefit of the patient welfare, and the hospital as an organization denoting in this way the significance of evidence-based medicine ${ }^{15,17}$ Evidence has also shown that "the greater the strength of the evidence incorporated, the greater the quality of the guideline and the greater its potential to maximize the use of resources and improve quality of healthcare" ${ }^{18}$.

\section{CLINICAL PROTOCOL OF LUNG TISSUE RESECTION}

Responsible staff: S-member of the surgical team, A-anesthesiologist, DN-department nurse, AN-anesthesiology nurse, $\mathrm{SN}$-surgical ward nurse, Phys-physical therapist

\section{Preoperative phase}

-Is adequate laboratory control available?

(Blood count, biochemical control, EKG, chest X ray, bronchoscopy, biopsies, brain CT, Abdomen CT, bones scan) $\mathbf{S}$

\begin{tabular}{|l|l|l|l|l|l|}
\hline Signature & Signature & Signature & Signature & Signature & Signature \\
\hline & & & & & \\
\hline
\end{tabular}
\begin{tabular}{|l|l|l|l|l|l|}
\hline Signature & Signature & Signature & Signature & Signature & Signature \\
\hline & & & & & \\
\hline
\end{tabular}

-Check for the procedure indication $\mathbf{S}$

\begin{tabular}{|l|l|l|l|l|l|}
\hline Signature & Signature & Signature & Signature & Signature & Signature \\
\hline & & & & & \\
\hline
\end{tabular}

-Antiplatelet antithrombotic drug interruption S

\begin{tabular}{|l|l|l|l|l|l|}
\hline Signature & Signature & Signature & Signature & Signature & Signature \\
\hline & & & & & \\
\hline
\end{tabular}

-Control for allergies S

\begin{tabular}{|l|l|l|l|l|l|}
\hline Signature & Signature & Signature & Signature & Signature & Signature \\
\hline & & & & & \\
\hline
\end{tabular}

-Patient preparation: enema, bath, shaving S, DN

\begin{tabular}{|l|l|l|l|l|l|}
\hline Signature & Signature & Signature & Signature & Signature & Signature \\
\hline & & & & & \\
\hline
\end{tabular}

-Is the patient diabetic? Remedy adjustment $\mathbf{S}$

\begin{tabular}{|l|l|l|l|l|l|}
\hline Signature & Signature & Signature & Signature & Signature & Signature \\
\hline & & & & & \\
\hline
\end{tabular}


-Are there any other comorbidities (e.g. thyroid disease); Remedy adjustment, consultation

S

\begin{tabular}{|l|l|l|l|l|l|}
\hline Signature & Signature & Signature & Signature & Signature & Signature \\
\hline & & & & & \\
\hline
\end{tabular}

\section{Operation Day}

-Patient identity check S, DN, AN, SN

\begin{tabular}{|l|l|l|l|l|l|}
\hline Signature & Signature & Signature & Signature & Signature & Signature \\
\hline & & & & & \\
\hline
\end{tabular}

-Confirmation of the kind of procedure S, A

\begin{tabular}{|l|l|l|l|l|l|}
\hline Signature & Signature & Signature & Signature & Signature & Signature \\
\hline & & & & & \\
\hline
\end{tabular}

-Removal of false teeth, rings, other metal objects DN, AN

\begin{tabular}{|l|l|l|l|l|l|}
\hline Signature & Signature & Signature & Signature & Signature & Signature \\
\hline & & & & & \\
\hline
\end{tabular}
Blood units availability check S/A
\begin{tabular}{|l|l|l|l|l|l|}
\hline Signature & Signature & Signature & Signature & Signature & Signature \\
\hline & & & & & \\
\hline
\end{tabular}

-Special care augmented alert for cases with difficult intubation A

\begin{tabular}{|l|l|l|l|l|l|}
\hline Signature & Signature & Signature & Signature & Signature & Signature \\
\hline & & & & & \\
\hline
\end{tabular}

\section{Postoperative phase}

- Is the patient suitable for fast track extubation S/A

\begin{tabular}{|l|l|l|l|l|l|}
\hline Signature & Signature & Signature & Signature & Signature & Signature \\
\hline & & & & & \\
\hline
\end{tabular}

-Continuous monitoring, regulation of fluid electrolytes balance-regulation of antibiotic and analgesic treatment-augmented clinical suspicion for postoperative bleeding S, Phys

\begin{tabular}{|l|l|l|l|l|l|}
\hline Signature & Signature & Signature & Signature & Signature & Signature \\
\hline & & & & & \\
\hline
\end{tabular}

$-1^{\text {st }}$ postoperative day, transport to the ward, removal of urine catheter $\mathbf{S}$ patient should sit and stand, respiratory gymnastics S, Phys

\begin{tabular}{|l|l|l|l|l|l|}
\hline Signature & Signature & Signature & Signature & Signature & Signature \\
\hline & & & & & \\
\hline
\end{tabular}


-Initiation of treatment with low molecular weight heparin, initation of treatment with antiplatelet drugs (SalospirClopidogrel), if indicated. -oral adjustment of drugs $\mathbf{S}$

\begin{tabular}{|l|l|l|l|l|l|}
\hline Signature & Signature & Signature & Signature & Signature & Signature \\
\hline & & & & & \\
\hline
\end{tabular}

$-2^{\text {nd }}-4^{\text {th }}$ postoperative day mobilization and chest tube removal

\begin{tabular}{|l|l|l|l|l|l|}
\hline Signature & Signature & Signature & Signature & Signature & Signature \\
\hline & & & & & \\
\hline
\end{tabular}

$2 n d-4^{\text {th }}$ postoperative day- removal of central venous catheter $\mathbf{S}$

\begin{tabular}{|l|l|l|l|l|l|}
\hline Signature & Signature & Signature & Signature & Signature & Signature \\
\hline & & & & & \\
\hline
\end{tabular}

$3^{d}-7^{\text {th }}$ postoperative day- discharge according to the range of tissue excision (wedge resection, lobectomy, pneumonectomy) $\mathbf{S}$

\begin{tabular}{|l|l|l|l|l|l|}
\hline Signature & Signature & Signature & Signature & Signature & Signature \\
\hline & & & & & \\
\hline
\end{tabular}

\section{Complications-Special Remarks}

\section{CLINICAL INDICATORS}

30 days mortality, hospitalization duration, readmission rate, reoperation rate

\section{CLINICAL PROTOCOL OF INGUINAL HERNIA PLASTIC OPERATION}

Responsible staff: S-member of the surgical team, A-anesthesiologist, DN-department nurse, AN-anesthesiology nurse, SN-surgical ward nurse, Phys-physical therapist

\section{Preoperative phase}

-Is adequate laboratory control available?

(Blood count, biochemical control, EKG, chest $\mathrm{X}$ ray) $\mathrm{S}$

\begin{tabular}{|l|l|l|l|l|l|}
\hline Signature & Signature & Signature & Signature & Signature & Signature \\
\hline & & & & & \\
\hline
\end{tabular}

-Is signed informed consent available $\mathbf{S}$

\begin{tabular}{|l|l|l|l|l|l|}
\hline Signature & Signature & Signature & Signature & Signature & Signature \\
\hline & & & & & \\
\hline
\end{tabular}

-Check for the procedure indication $\mathbf{S}$

\begin{tabular}{|l|l|l|l|l|l|}
\hline Signature & Signature & Signature & Signature & Signature & Signature \\
\hline & & & & & \\
\hline
\end{tabular}

-Antiplatelet antithrombotic drug interruption $\mathbf{S}$

\begin{tabular}{|l|l|l|l|l|l|}
\hline Signature & Signature & Signature & Signature & Signature & Signature \\
\hline & & & & & \\
\hline
\end{tabular}

-Control for allergies S 


\begin{tabular}{|l|l|l|l|l|l|}
\hline Signature & Signature & Signature & Signature & Signature & Signature \\
\hline & & & & & \\
\hline
\end{tabular}

-Patient preparation: enema, bath, shaving S, DN

\begin{tabular}{|l|l|l|l|l|l|}
\hline Signature & Signature & Signature & Signature & Signature & Signature \\
\hline & & & & & \\
\hline
\end{tabular}

-Is the patient diabetic? Remedy adjustment $\mathbf{S}$

\begin{tabular}{|l|l|l|l|l|l|}
\hline Signature & Signature & Signature & Signature & Signature & Signature \\
\hline & & & & & \\
\hline
\end{tabular}

-Are there any other comorbidities? (e.g. thyroid disease); Remedy adjustment, consultation

S

\begin{tabular}{|l|l|l|l|l|l|}
\hline Signature & Signature & Signature & Signature & Signature & Signature \\
\hline & & & & & \\
\hline
\end{tabular}

- Is mesh graft available? $\mathbf{S}$

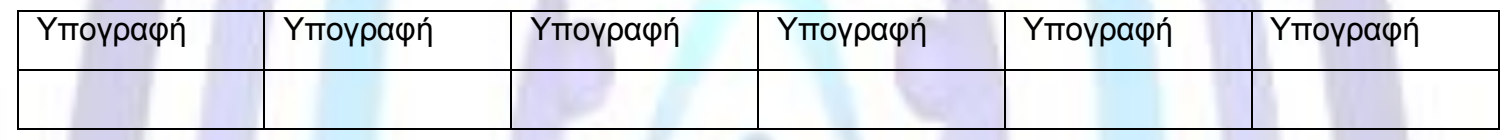

Patient identity check S, DN, AN, SN

\begin{tabular}{|l|l|l|l|l|l|}
\hline Signature & Signature & Signature & Signature & Signature & Signature \\
\hline & & & & & \\
\hline
\end{tabular}

-Confirmation of the kind and side of procedure S, A

\begin{tabular}{|l|l|l|l|l|l|}
\hline Signature & Signature & Signature & Signature & Signature & Signature \\
\hline & & & & & \\
\hline
\end{tabular}

-Removal of false teeth, rings, other metal objects DN, AN

\begin{tabular}{|l|l|l|l|l|l|}
\hline Signature & Signature & Signature & Signature & Signature & Signature \\
\hline & & & & & \\
\hline
\end{tabular}

- Blood products availability check S, A

\begin{tabular}{|l|l|l|l|l|l|}
\hline Signature & Signature & Signature & Signature & Signature & Signature \\
\hline & & & & & \\
\hline
\end{tabular}

-Special care and augmented alert for cases with difficult intubation A

\begin{tabular}{|l|l|l|l|l|l|}
\hline Signature & Signature & Signature & Signature & Signature & Signature \\
\hline & & & & & \\
\hline
\end{tabular}

\section{Postoperative phase}

-Immediate extubation, transfer to the ward A, $\mathbf{S}$ 


\begin{tabular}{|l|l|l|l|l|l|}
\hline Signature & Signature & Signature & Signature & Signature & Signature \\
\hline & & & & & \\
\hline
\end{tabular}

-Operation day, Regulation of antibiotic and analgesic treatment, mobilization S, Phys

\begin{tabular}{|l|l|l|l|l|l|}
\hline Signature & Signature & Signature & Signature & Signature & Signature \\
\hline & & & & & \\
\hline
\end{tabular}

-Initiation of treatment with low molecular weight heparin, initiation of treatment with antiplatelet drugs, adjustment of other regimens $\mathbf{S}$

\begin{tabular}{|l|l|l|l|l|l|l|}
\hline Signature & Signature & Signature & Signature & Signature & Signature & Signature \\
\cline { 1 - 7 } & & & & & & \\
\cline { 1 - 6 }
\end{tabular}

-Day of the operation or postoperative day 1 Control of the surgical wound, removal of drainage, discharge $S$

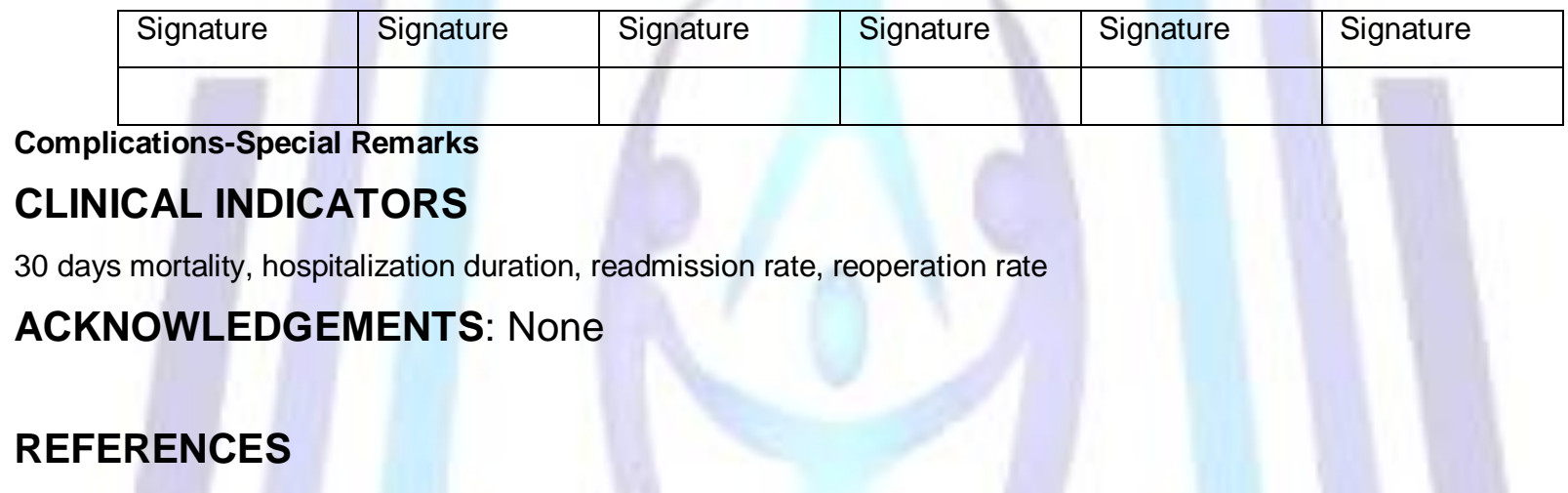

1. Tagarakis G, Dikeos C, Tsolaki F, Daskalopoulos M, Bougioukakis P, Tsilimingas N, Polyzos N. The significance of clinical protocols in surgical disciplines. Journal of Social Sciences Research. Vol. 6, No 1, Nov. 2014

2. Varela G, Thomas PA. Surgical management of advanced non-small cell lung cancer. J Thorac Dis. 2014 May;6(Suppl2):S217-23

3. Moeller A, Sartipy U. Predictors of postoperative quality of life after surgery for lung cancer. J Thorac Oncol. 2012 Feb;7(2):406-11

4. Nagamatsu Y, Iwasaki Y, Hayashida R, Kashihara M, Yoshiyama K, Yamana H, Shirouzu K. Factors related to an early restoration of exercise capacity after major lung resection. Surg Today. 2011 Sep;41(9):1228-33

5. De Ruysscher D, Nakagawa K, Asamura H. Surgical and nonsurgical approaches to small-size non small cell lung cancer. Eur Respir J. 2014 Jun 12

6. Rick O, Metz T, Eberlein M, Schirren J, Bolukbas S. The Six-Minute-Walk Test in assessing respiratory function after tumor surgery of the lung: a cohort study. J Thorac Dis. 2014 May;6(5):421-8

7. Bjurstrom MF, Nicol Al, Amid PK, Chen DC. Pain control following inguinal herniorrhaphy: current perspectives. Pain Res. 2014 May 29;7:277-90

8. Compagna R, Vigliotti G, Bianco T, Amato M, Rossi R, Fappiano F, Accurso A, Danzi M, Aprea G, Amato B. Local anesthesia for treatment of hernia in elder patients: Levobupicavaine or Bupivacaine? BMC Surg. 2013;13 Suppl 2:S30

9. Shyam DC, Rapsang AG. Inguinal hernias in patients of 50 years and above. Pattern and outcome. Rev Col Bras Cir. 2013 Sep-Oct;40(5):374-9

10. Odobasic A, Krdzalic G, Hodzic M, Hasukic S, Sehanovic A, Odobasic A. The role of fibrin glue polypropylene mesh fixation in open inguinal hernia repair. Med Arh. 2014;68(2):90-3 


\section{ISSN 2321-1091}

11. Gys $T$, Gys B, Lafullarde $T$. The use of a self-gripping mesh in open inguinal hernia repair. A prospective observational single surgeon study. Acta Chir Belg. 2013 May-Jun;113():192-5

12. Akcaboy EY, Akcaboy ZN, Gogus N. Ambulatory inguinal herniorrhaphy: paravertebral block versus spinal anesthesia. Minerva Anestesiol. 2009 Dec;75(12):684-91

13. Polyzos N, Karanikas H, Thireos E, Kastanioti C, Kontodimopoulos N. Reforming reimbursement of public hospitals in Greece during the economic crisis: Implementation of a DRG system. Health Policy. 2013 Jan;109(1):14-22

14. Bahtsevani C, Udén G, Willman A. Outcomes of evidence-based clinical practice guidelines: a systematic review. International journal of technology assessment in healthcare. 2004 Nov;20(04): 427-433.

15. Machikanti L, Evidence-Based Medicine, Systematic Reviews, and Guidelines in Interventional Pain Management, Part I: Introduction and General Considerations

16. Woolf S, Schünemann H, Eccles M, Grimshaw J, Shekelle P, Developing Clinical Practice Guidelines: types of evidence and outcomes values and economics, synthesis, grading, and presentation and deriving recommendations. Implementation Science, 2012 Jul;7(61) 1-12

17. Weisz G, Cambrosio A, Keating P, Knaapen L, Schlich T, Tournay V. The emergence of clinical practice guidelines. Milbank Quarterly. 2007 Dec;85(4), 691-727.

18. Knai C, Brusamento S, Legido-Quigley H, Saliba V, Panteli D, Turk E, et al. Systematic review of the methodological quality of clinical guideline development for the management of chronic disease in Europe. Health Policy. 2012 Oct;107(2):157-167. 